Supporting Information

\title{
Printable High-voltage Integrated Micro-Supercapacitors based on Heteroatom-doped Porous Biomass Carbon
}

Zhangyi Xiongt, Peijing Guoł, Shaoyu Yuant, Sufang Sunt, Chun Wangł* and Yongjun Gao ${ }^{*} *$

$\dagger$ Key Laboratory of Medicinal Chemistry and Molecular Diagnosis of Ministry of Education, Key Laboratory of Analytical Science and Technology of Hebei Province, College of Chemistry and Environmental Science, Hebei University, Baoding, 071002 China

$\$$ College of Science, Hebei Agricultural University, Baoding, 071001, China

*Corresponding author,

E-mail: yjgao@hbu.edu.cn (Yongjun Gao),

E-mail: wangchun@hebau.edu.cn (Chun Wang). 


\section{content}

Figure S1. (a) XRD spectra of KNSC-T-3. (b) Raman spectra of KNSC-T-3 ............................

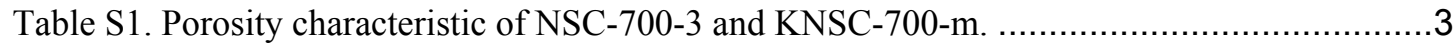

Figure $\mathrm{S} 2$. (a) $\mathrm{N}_{2}$ adsorption-desorption isotherm curves of KNSC- $T$-3. (b) the pore-size distribution

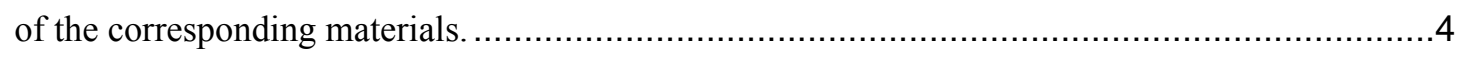

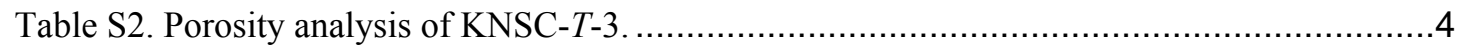

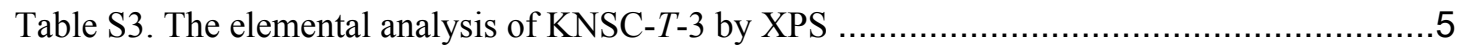

Figure S3. The XPS survey spectrum of NSC-700-3 and KNSC-700-3 ...............................

Figure S4. (a) XPS C1s spectra of NSC-700-3 and KNSC-700-3. (b) XPS O1s spectra of NSC700-3 and KNSC-700-3.

Figure S5. XPS spectra of KNSC-T-3. (a) C 1s. (b) N 1s. (c) O 1s. (d) S 2p...........................

Figure S6. Electrochemical performance under different carbonization temperature. (a) CV curves of KNSC-700-3 at different scan rate. (b) CV curves of NSC-700-3 at different scan rate. ...........8

Table S4. Summary and comparison of electrochemical performance for carbon-based materials. 9 Figure S7. (a) GCD profiles of KNSC-700-3 at different current densities. (b) specific capacitance of KNSC-700-3 as a function of current density in $1 \mathrm{M} \mathrm{H}_{2} \mathrm{SO}_{4}$ electrolyte. 10

Figure S8. (a) CV curves of NSC-700-3 with separated contribution of capacitive (red area) and pseudo-capacitive (white area) capacitance at $20 \mathrm{mV} \mathrm{s}^{-1}$. (b) Percentage stacked histogram of NSC700-3 show the contribution of EDLC and pseudo-capacitance at various scan rate. .................10

Figure S9. The equivalent electric circuit used for fitting the EIS spectra.............................11

Table S5. Fitting results of NSC-700-3 and KNSC-700-m by EIS spectra.............................11

Figure S10. (a) Bode plots of NSC-700-3 and KNSC-700-m. (b) The histogram of time constant $\tau_{0}$ for corresponding materials. 12

Figure S11. (a) CV curves of KNSC-T-3 at a scan rate of $20 \mathrm{mV} \mathrm{s}^{-1}$. (b) GCD profiles of 
corresponding KNSCs materials at a current density of $1 \mathrm{~A} \mathrm{~g}^{-1}$. (c) Nyquist plots of KNSC-T-3. (d)

Bode plots of KNSC-T-3. (e) The histogram of time constant $\tau_{0}$ for KNSC-T-3.

Figure S12. The parameters of micro-supercapacitors size for various geometries pattern including

(a) interdigital, (b) linear shapes

Table S6. Summary and comparison of electrochemical performance for selected MSCs. 15
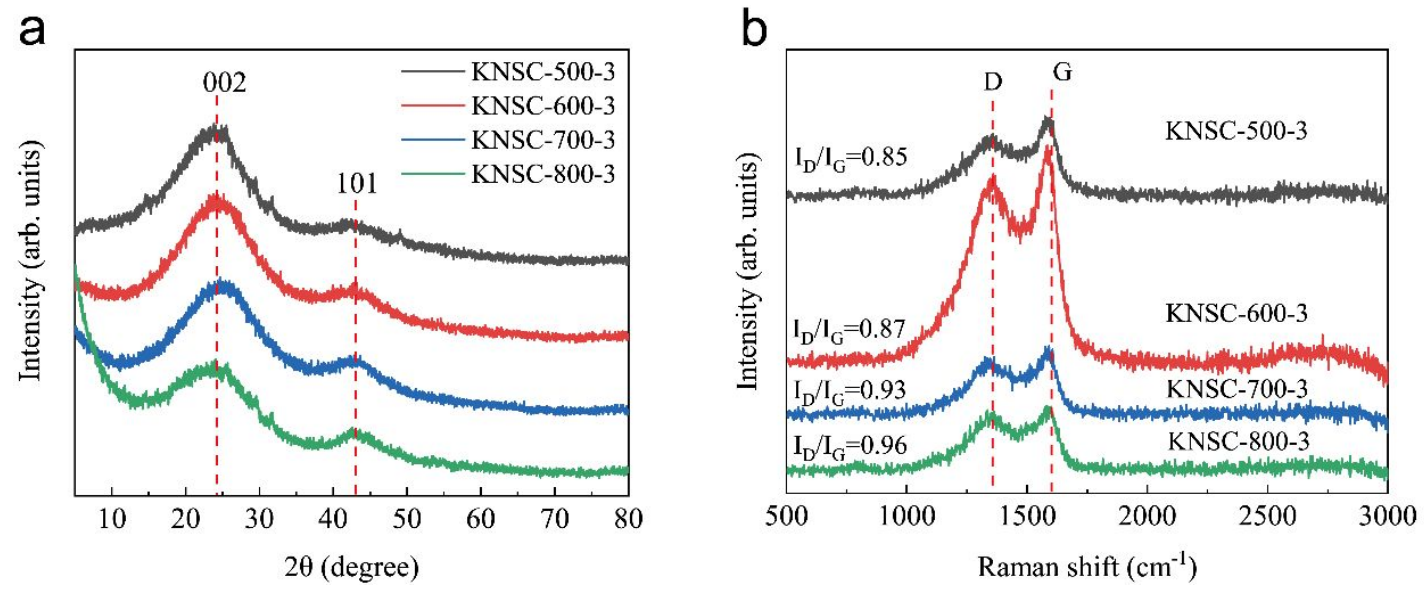

Figure S1. (a) XRD spectra of KNSC-T-3. (b) Raman spectra of KNSC-T-3.

Table S1. Porosity characteristic of NSC-700-3 and KNSC-700-m.

\begin{tabular}{ccccc}
\hline sample & $\mathbf{S}_{\text {BET }} / \mathbf{m}^{\mathbf{2}} \mathbf{g}^{-\mathbf{1}}$ & $\mathbf{V}_{\text {mes }} / \mathbf{c m}^{\mathbf{3}} \mathbf{g}^{-\mathbf{1}}$ & $\mathbf{V}_{\text {total }} / \mathbf{c m}^{\mathbf{3}} \mathbf{g}^{-\mathbf{1}}$ & $\mathbf{V}_{\text {mes }} / \mathbf{V}_{\text {total }} / \mathbf{\%}$ \\
\hline NSC-700-3 & 54 & 0.029 & 0.044 & 66 \\
KNSC-700-1 & 1323 & 0.279 & 0.722 & 39 \\
KNSC-700-2 & 1423 & 0.435 & 0.866 & 50 \\
\hline
\end{tabular}




\begin{tabular}{ccccc}
\hline KNSC-700-3 & 1689 & 0.215 & 0.886 & 24 \\
KNSC-700-4 & 2116 & 0.205 & 1.093 & 19 \\
\hline
\end{tabular}

a

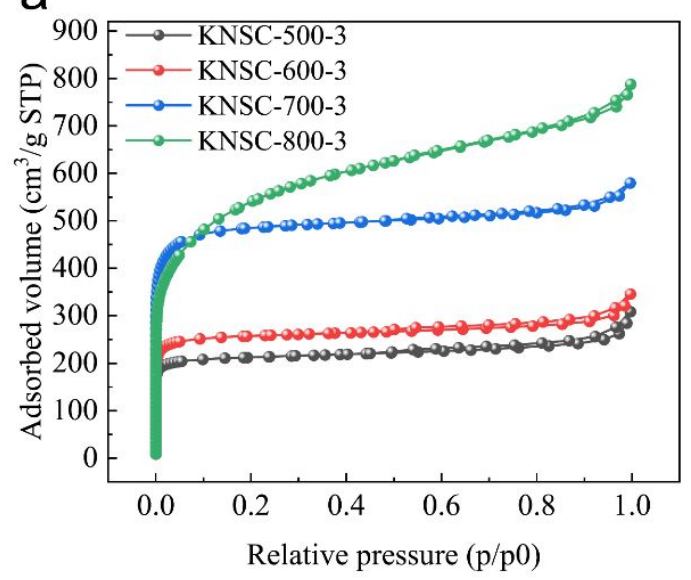

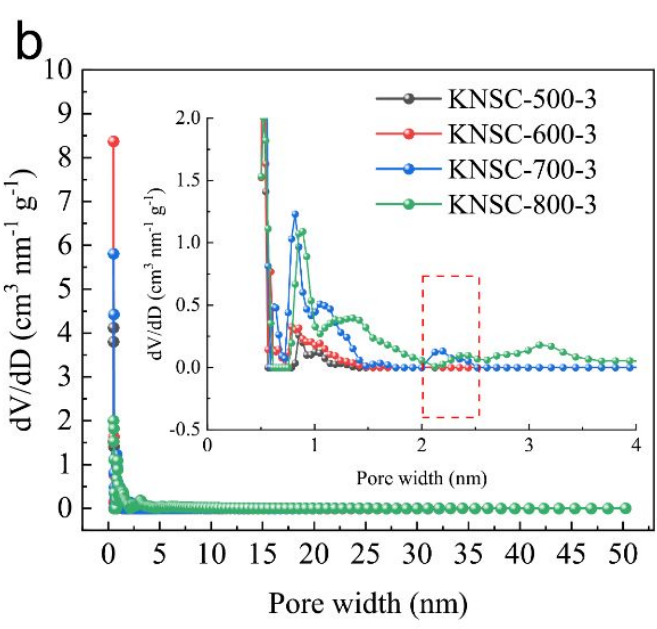

Figure S2. (a) $\mathrm{N}_{2}$ adsorption-desorption isotherm curves of KNSC-T-3. (b) the poresize distribution of the corresponding materials.

Table S2. Porosity analysis of KNSC-T-3.

\begin{tabular}{ccccc}
\hline sample & $\mathbf{S}_{\text {BET }} / \mathbf{m}^{\mathbf{2}} \mathbf{g}^{-1}$ & $\mathbf{V}_{\mathbf{m e}} / \mathbf{c m}^{\mathbf{3}} \mathbf{g}^{-1}$ & $\mathbf{V}_{\text {tota }} \mathbf{l} / \mathbf{c m}^{\mathbf{3}} \mathbf{g}^{-}$ & \\
\hline KNSC-500-3 & 739 & 0.179 & 0.445 & 40 \\
KNSC-600-3 & 897 & 0.174 & 0.514 & 34 \\
KNSC-700-3 & 1689 & 0.215 & 0.886 & 24 \\
\hline
\end{tabular}




\begin{tabular}{lllll}
\hline KNSC-800-3 & 1881 & 0.753 & 1.185 & 64 \\
\hline
\end{tabular}

Table S3. The elemental analysis of KNSC-T-3 by XPS

\begin{tabular}{ccccc}
\hline sample & C(at\%) & N(at\%) & O(at\%) & S(at\%) \\
\hline NSC-700-3 & 81.01 & 1.61 & 15.55 & 1.82 \\
KNSC-500-3 & 87.89 & 1.72 & 7.97 & 2.42 \\
KNSC-600-3 & 91.64 & 1.59 & 6.06 & 0.7 \\
KNSC-700-3 & 91.46 & 1.43 & 6.25 & 0.86 \\
KNSC-800-3 & 90 & 1.2 & 7.5 & 1.31 \\
\hline
\end{tabular}

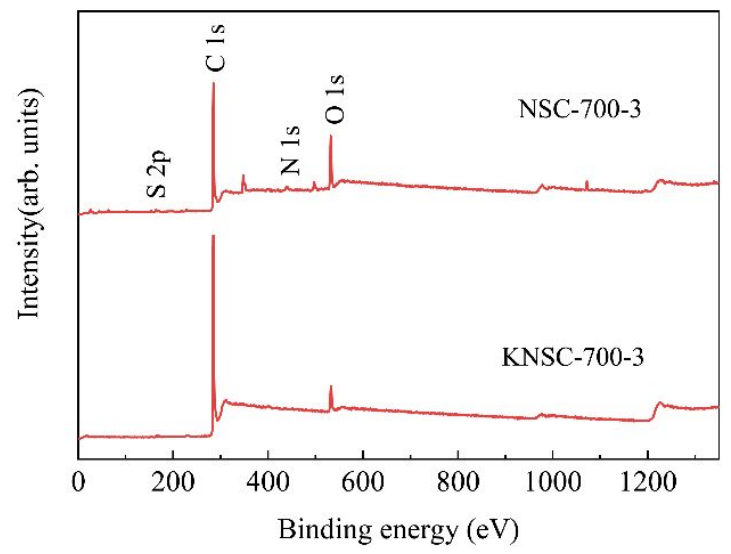

Figure S3. The XPS survey spectrum of NSC-700-3 and KNSC-700-3. 

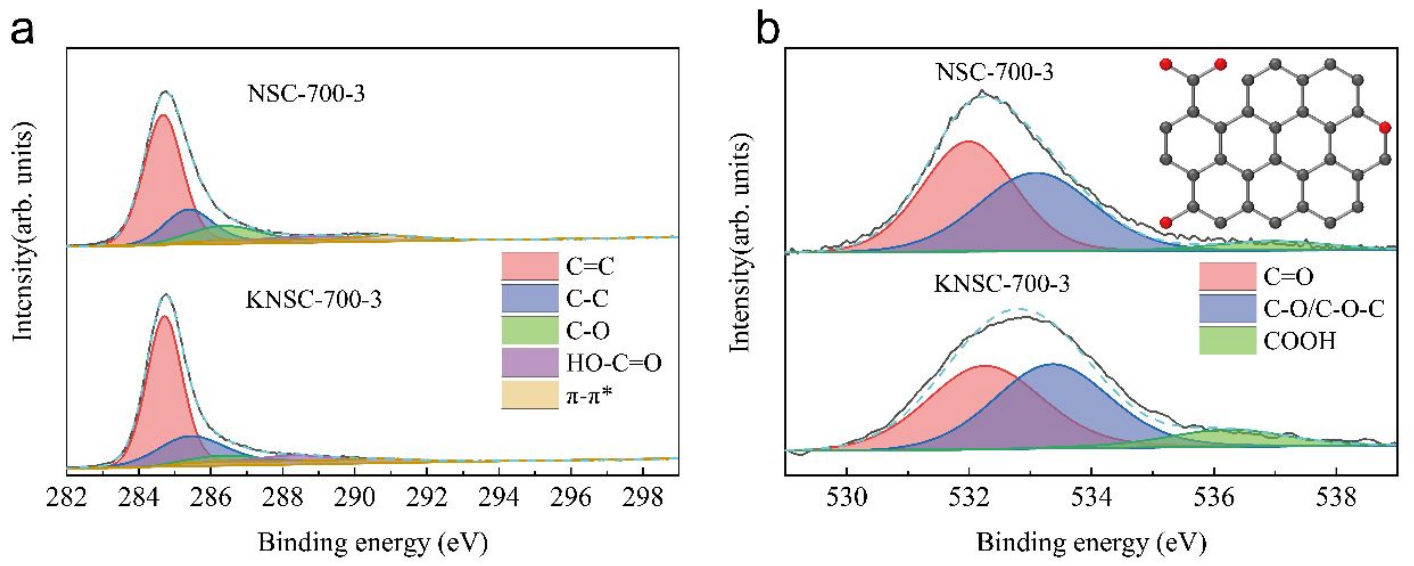

Figure S4. (a) XPS C1s spectra of NSC-700-3 and KNSC-700-3. (b) XPS O1s spectra of NSC-700-3 and KNSC-700-3. 

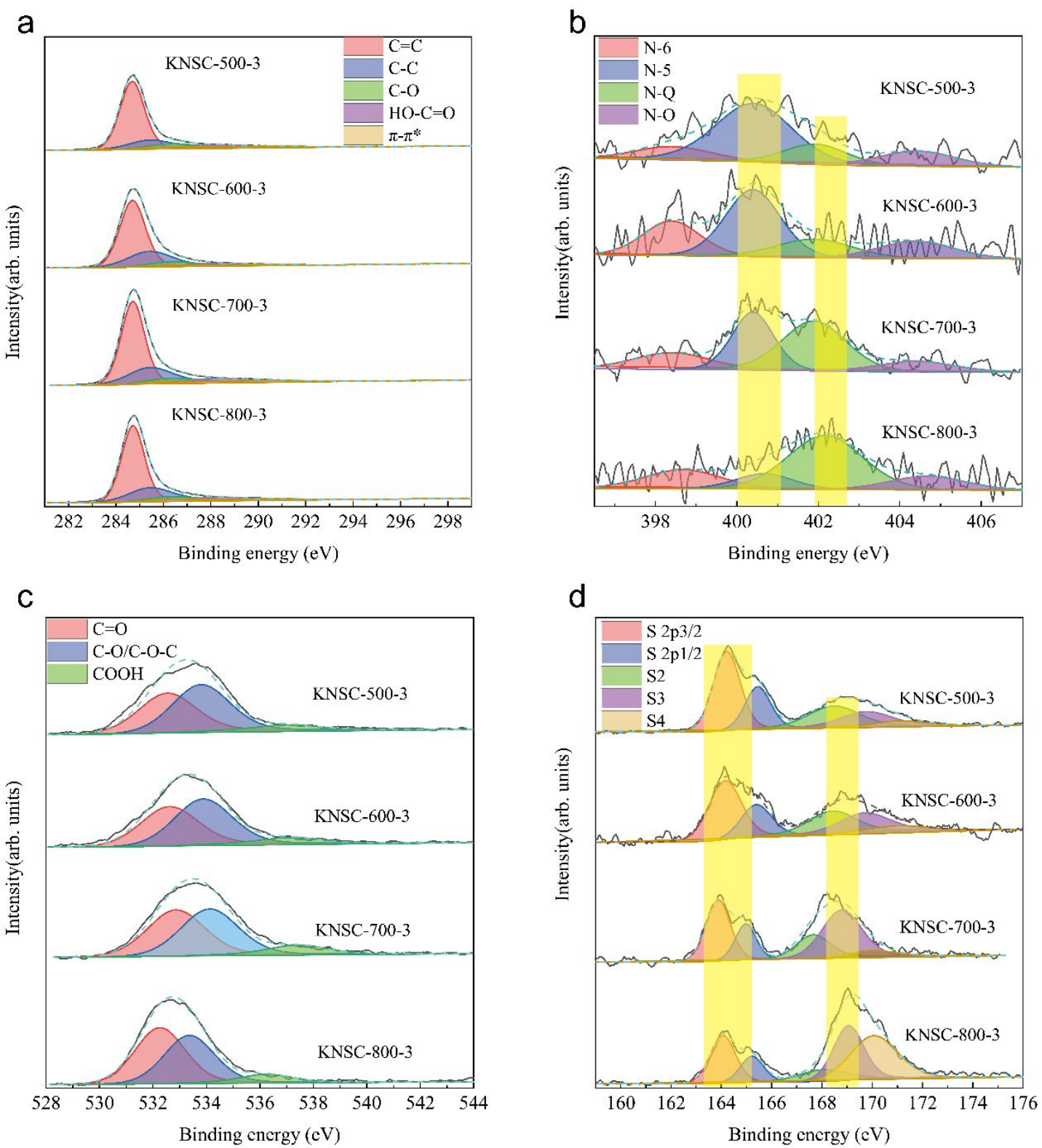

Figure S5. XPS spectra of KNSC-T-3. (a) C 1s. (b) N 1s. (c) O 1s. (d) S 2p. 

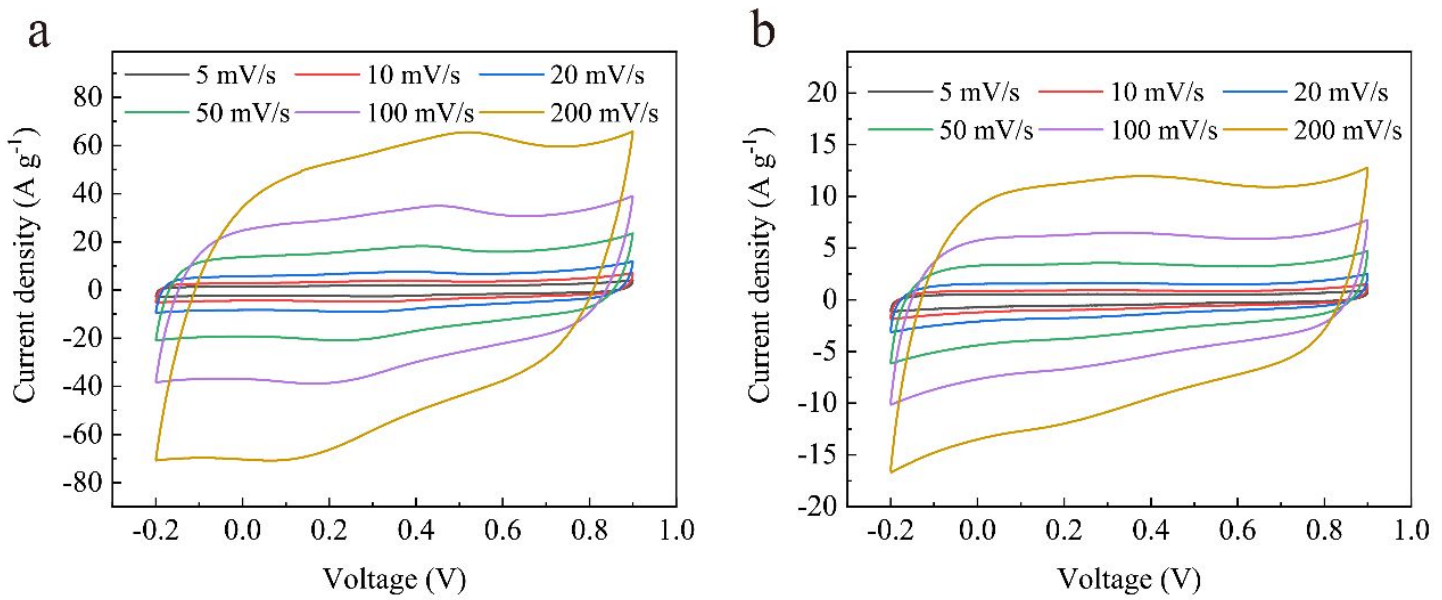

Figure S6. Electrochemical performance under different carbonization temperature. (a)

CV curves of KNSC-700-3 at different scan rate. (b) CV curves of NSC-700-3 at different scan rate. 
Table S4. Summary and comparison of electrochemical performance for carbon-based materials.

\begin{tabular}{|c|c|c|c|c|c|c|}
\hline materials & Electrolyte & $\begin{array}{l}\text { Current } \\
\text { density } \\
\left(\mathrm{A} \mathrm{g}^{-1}\right)\end{array}$ & $\begin{array}{c}\text { Specific } \\
\text { capacitance } \\
\left(\mathrm{F} \mathrm{g} \mathrm{g}^{-1}\right)\end{array}$ & $\begin{array}{c}\text { Energy } \\
\text { density } \\
\left(\mathrm{Wh} \mathrm{kg}^{-1}\right)\end{array}$ & $\begin{array}{c}\text { Power } \\
\text { density } \\
\text { (W kg- } \\
1 \text { ) }\end{array}$ & Ref \\
\hline N-doped carbon & $\mathrm{H}_{2} \mathrm{SO}_{4}$ & 0.5 & 346 & 11.9 & 2995 & 1 \\
\hline $\begin{array}{c}\text { hollow mesoporous } \\
\text { carbon spheres }\end{array}$ & $\mathrm{KOH}$ & 0.5 & 429 & 47.8 & 17112 & 2 \\
\hline $\begin{array}{c}\text { nitrogen-doped graphite } \\
\text { oxide }\end{array}$ & $\mathrm{H}_{2} \mathrm{SO}_{4}$ & 0.5 & 201 & 11 & 278 & 3 \\
\hline Nitrogen carbon sphere & $\mathrm{KOH}$ & 0.1 & 276 & 7.8 & 6200 & 4 \\
\hline bagasse-based carbon & $\mathrm{KOH}$ & 1 & 413 & 22.3 & 9000 & 5 \\
\hline $\begin{array}{l}\text { nitrogen doped porous } \\
\text { nanorods }\end{array}$ & $\mathrm{KOH}$ & 1.25 & 162 & -- & -- & 6 \\
\hline $\begin{array}{l}\text { N/S dual-doped carbon } \\
\text { fibers }\end{array}$ & $\mathrm{KOH}$ & 1 & 202 & -- & -- & 7 \\
\hline KNSC-700-3 & $\mathrm{H}_{2} \mathrm{SO}_{4}$ & 1 & 481 & 16.5 & 5091 & $\begin{array}{l}\text { This } \\
\text { work }\end{array}$ \\
\hline
\end{tabular}



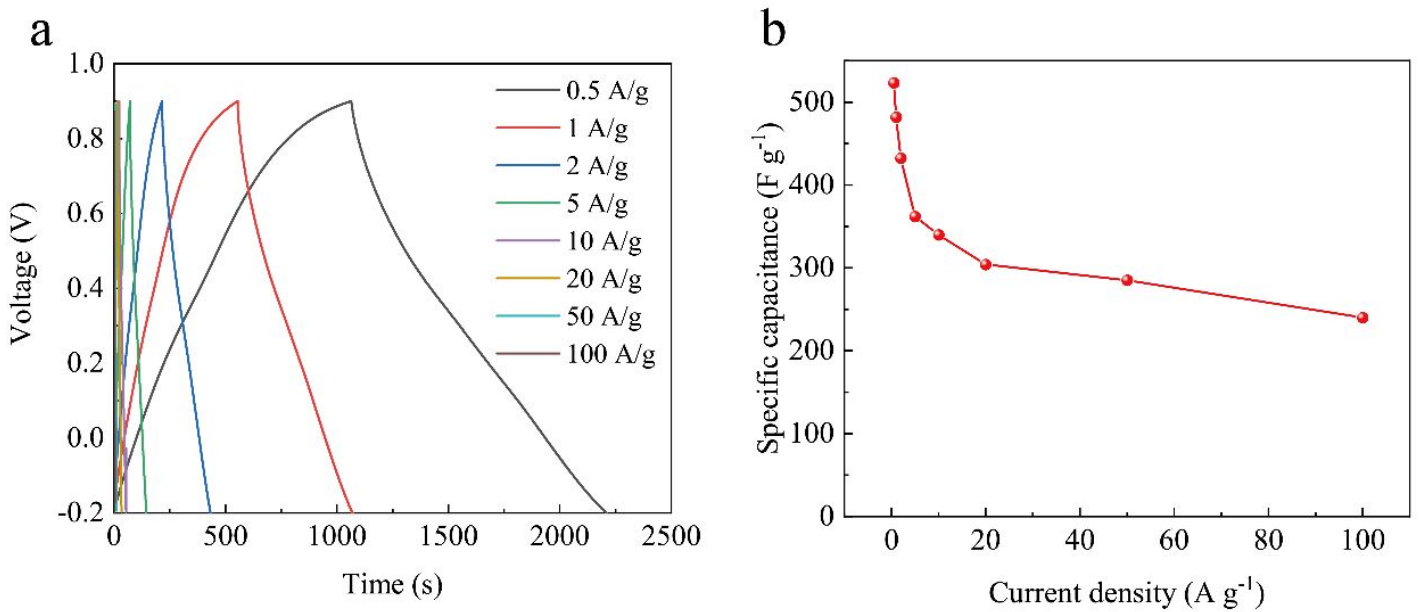

Figure S7. (a) GCD profiles of KNSC-700-3 at different current densities. (b) specific capacitance of KNSC-700-3 as a function of current density in $1 \mathrm{M} \mathrm{H}_{2} \mathrm{SO}_{4}$ electrolyte.
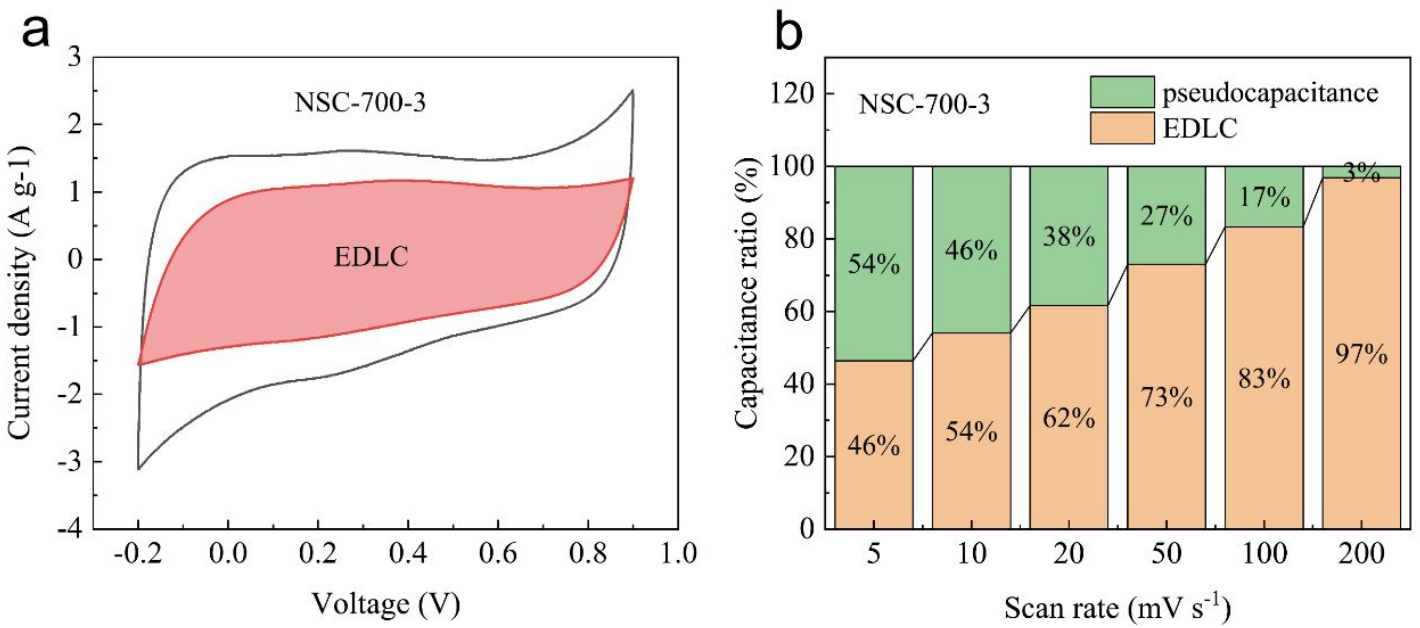

Figure S8. (a) CV curves of NSC-700-3 with separated contribution of capacitive (red area) and pseudo-capacitive (white area) capacitance at $20 \mathrm{mV} \mathrm{s}^{-1}$. (b) Percentage stacked histogram of NSC-700-3 show the contribution of EDLC and pseudocapacitance at various scan rate. 


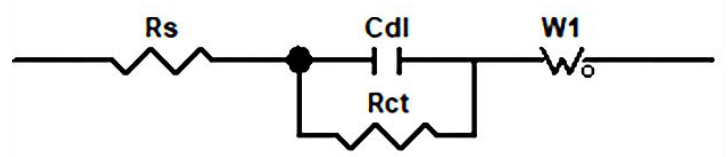

Figure S9. The equivalent electric circuit used for fitting the EIS spectra.

Table S5. Fitting results of NSC-700-3 and KNSC-700-m by EIS spectra.

\begin{tabular}{ccc}
\hline Sample & $\mathbf{R}_{\mathbf{s}} / \mathbf{\Omega}$ & $\mathbf{R}_{\mathbf{c t}} / \mathbf{\Omega}$ \\
\hline NSC-700-3 & 0.4935 & 0.8312 \\
KNSC-700-1 & 0.4910 & 0.7361 \\
KNSC-700-2 & 0.4494 & 0.8274 \\
KNSC-700-3 & 0.3799 & 0.4814 \\
KNSC-700-4 & 0.4456 & 0.5344 \\
\hline
\end{tabular}


a

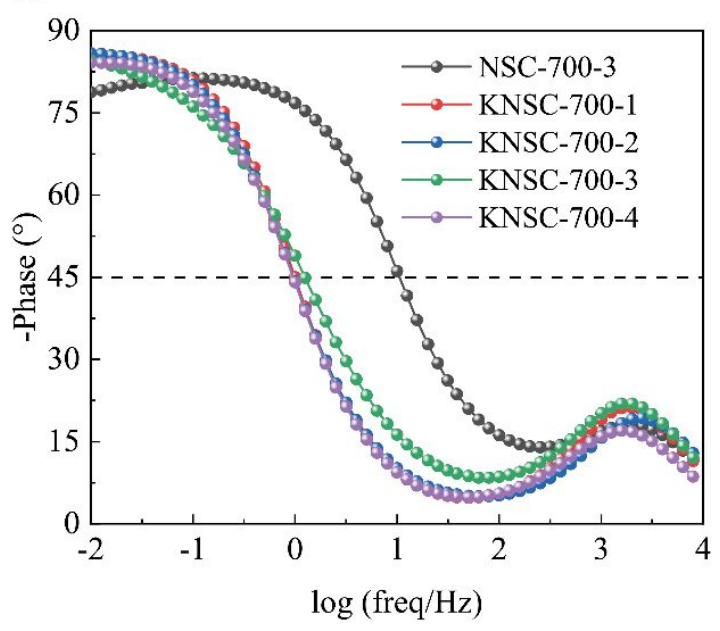

b

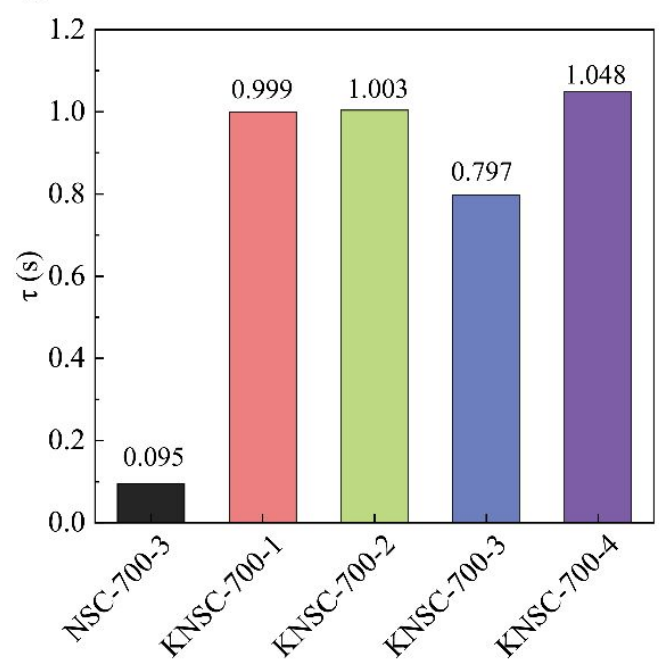

Figure S10. (a) Bode plots of NSC-700-3 and KNSC-700-m. (b) The histogram of time constant $\tau_{0}$ for corresponding materials. 
a

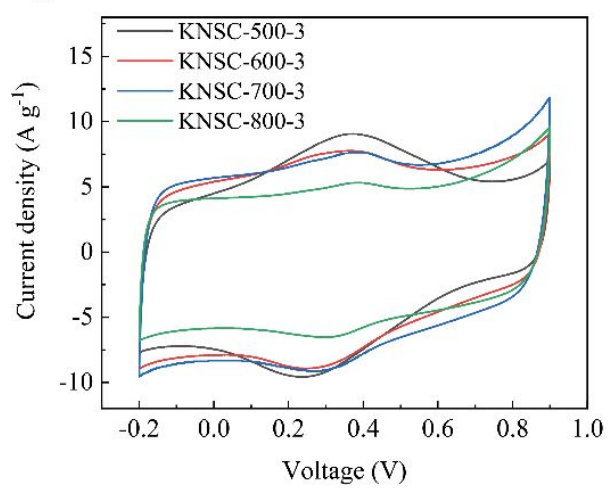

C

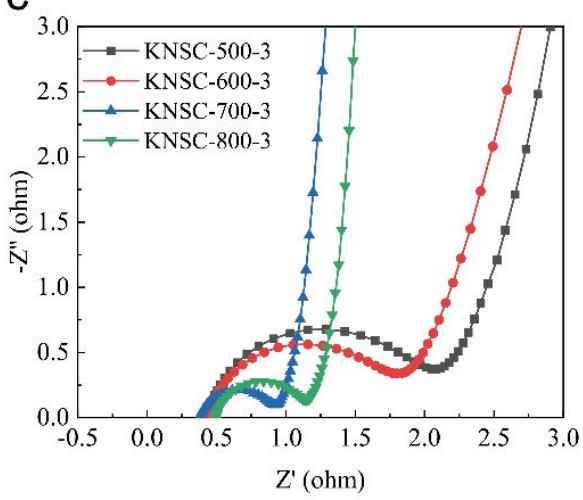

e

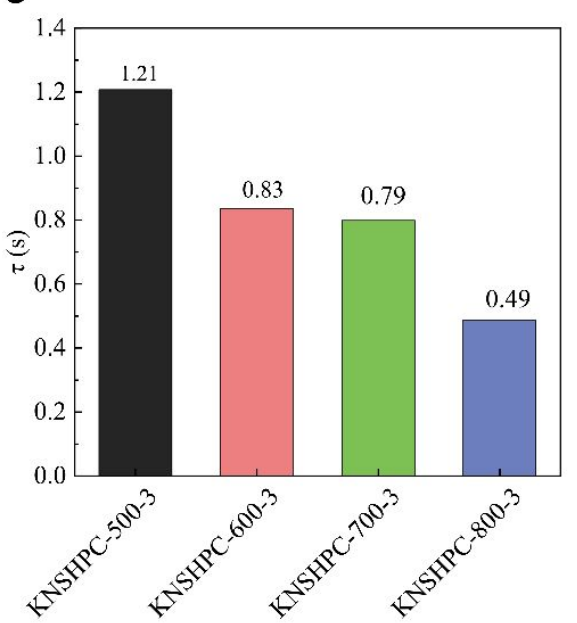

b

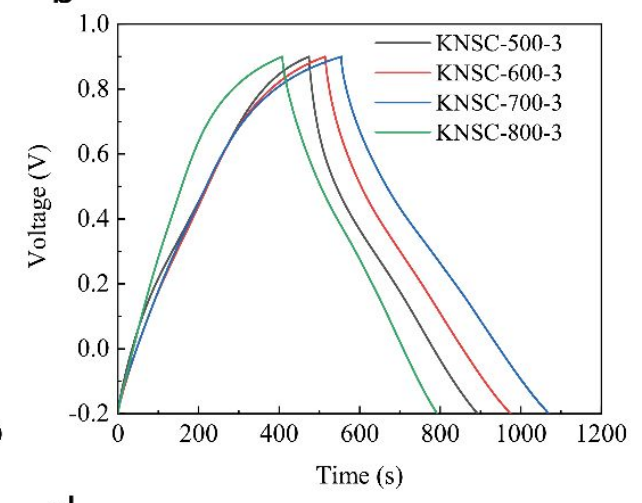

d

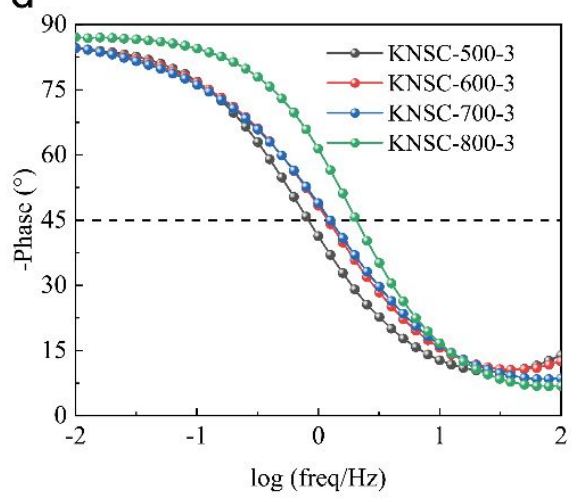

Figure S11. (a) CV curves of KNSC-T-3 at a scan rate of $20 \mathrm{mV} \mathrm{s}^{-1}$. (b) GCD profiles of corresponding KNSCs materials at a current density of $1 \mathrm{~A} \mathrm{~g}^{-1}$. (c) Nyquist plots of KNSC-T-3. (d) Bode plots of KNSC-T-3. (e) The histogram of time constant $\tau_{0}$ for KNSC-T-3. 

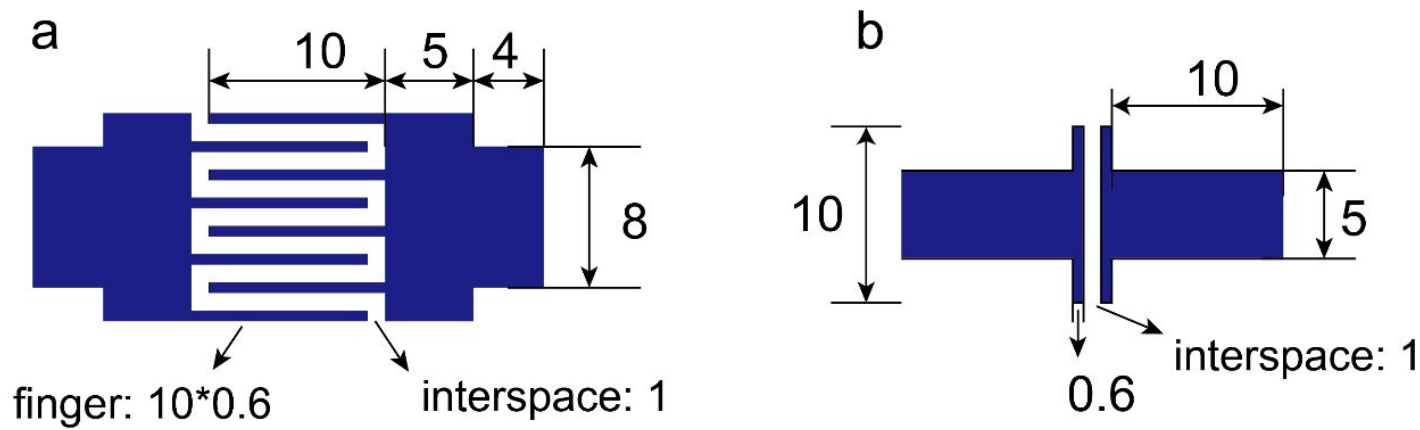

unit: $\mathrm{mm}$

Figure S12. The parameters of micro-supercapacitors size for various geometries pattern including (a) interdigital, (b) linear shapes. 
Table S6. Summary and comparison of electrochemical performance for selected MSCs.

\begin{tabular}{ccccccc}
\hline Supercapacitors & Electrolyte & $\begin{array}{c}\text { Potential } \\
(\mathrm{V})\end{array}$ & $\begin{array}{c}\text { Current } \\
\text { density }\end{array}$ & $\begin{array}{c}\text { Areal } \\
\text { capacitance } \\
\left(\mathrm{mF} \mathrm{cm}^{-2}\right)\end{array}$ & $\begin{array}{c}\text { Energy } \\
\text { density } \\
(\mathrm{mWh} \\
\left.\mathrm{cm}^{-3}\right)\end{array}$ & Ref \\
\hline EG/ $\mathrm{V}_{2} \mathrm{O}_{5}$-MSC & $\mathrm{LiCl} / \mathrm{PVA}$ & 1 & $0.02 \mathrm{~mA} \mathrm{~cm}^{-2}$ & 2.3 & 20 & 8 \\
GP-MSs & $\mathrm{H}_{2} \mathrm{SO}_{4} / \mathrm{PVA}$ & 0.8 & $2 \mathrm{mV} \mathrm{s}^{-1}$ & 4.9 & 1.4 & 9 \\
GP/PANI-G/GP & $\mathrm{H}_{2} \mathrm{SO}_{4} / \mathrm{PVA}$ & 0.8 & $2 \mathrm{mV} \mathrm{s}^{-1}$ & 7.6 & 3.1 & 9 \\
RArG-4-200 & $\mathrm{KOH} / \mathrm{PVA}_{\text {LIG-MSCs }}$ & 1 & $0.08 \mathrm{~mA} \mathrm{~cm}^{-2}$ & 10.4 & 18.8 & 10 \\
P3MT/ HACNT & $\mathrm{H}_{3} \mathrm{PO}_{4} / \mathrm{PVA}$ & 0.8 & $5 \mathrm{mV} \mathrm{s}^{-1}$ & 0.62 & 0.47 & 11 \\
interdigital & $\mathrm{Et}_{4} \mathrm{NBF}_{4} / \mathrm{PC}$ & 3.5 & $5 \mathrm{mV} \mathrm{s}^{-1}$ & 3.1 & 1.08 & 12 \\
IMSCs & $\mathrm{H}_{2} \mathrm{SO}_{4} / \mathrm{PVA}$ & 0.8 & $0.1 \mathrm{~mA} \mathrm{~cm}^{-2}$ & 13.6 & 0.6 & This \\
linear IMSCs & $\mathrm{H}_{2} \mathrm{SO}_{4} / \mathrm{PVA}$ & 8.8 & $1 \mathrm{~mA} \mathrm{~cm}^{-2}$ & 102.7 & 552.3 & work \\
\hline
\end{tabular}

\section{REFERENCES}

(1) Chu, M.; Zhai, Y.; Shang, N.; Guo, P.; Wang, C.; Gao, Y., N-doped carbon derived from the monomer of chitin for high-performance supercapacitor. Appl. Surf. Sci. 2020, $517,146140$.

(2) Du, J.; Liu, L.; Yu, Y.; Zhang, Y.; Lv, H.; Chen, A., Interpolation strategy for monodisperse hollow mesoporous carbon spheres in high performance supercapacitor. J. Power Sources 2019, 434, 226720.

(3) Ghanashyam, G.; Jeong, H. K., Synthesis of plasma treated nitrogen-doped graphite oxide for supercapacitor applications. J. Energy Storage 2019, 26, 100923.

(4) Pang, J.; Zhang, W.; Zhang, H.; Zhang, J.; Zhang, H.; Cao, G.; Han, M.; Yang, Y., Sustainable nitrogen-containing hierarchical porous carbon spheres derived from sodium lignosulfonate for high-performance supercapacitors. Carbon 2018, 132, 280 293. 
(5) Yu, P.; Liang, Y.; Dong, H.; Hu, H.; Liu, S.; Peng, L.; Zheng, M.; Xiao, Y.; Liu, Y., Rational Synthesis of Highly Porous Carbon from Waste Bagasse for Advanced Supercapacitor Application. ACS Sustain. Chem. Eng. 2018, 6, 15325-15332.

(6) Zhu, W.; Wang, H.; Zhao, R.; Yang, M.; Liu, Y.; Yan, D., In situ fabrication of nitrogen doped porous carbon nanorods derived from metal-organic frameworks and its application as supercapacitor electrodes. J. Solid State Chem. 2019, 277, 100-106.

(7) Lei, W.; Zhang, H.; Liu, D.; Lin, L., Fabrication of nitrogen and sulfur co-doped carbon nanofibers with three-dimensional architecture for high performance supercapacitors. Appl. Surf. Sci. 2019, 495, 143572.

(8) Zhang, P.; Zhu, F.; Wang, F.; Wang, J.; Dong, R.; Zhuang, X.; Schmidt, O. G.; Feng, X., Stimulus-Responsive Micro-Supercapacitors with Ultrahigh Energy Density and Reversible Electrochromic Window. Adv. Mater. 2017, 29, 1604491.

(9) Shi, X.; Wu, Z. S.; Qin, J.; Zheng, S.; Wang, S.; Zhou, F.; Sun, C.; Bao, X., Graphene-Based Linear Tandem Micro-Supercapacitors with Metal-Free Current Collectors and High-Voltage Output. Adv. Mater. 2017, 29, 1703034.

(10)Li, H.; Liu, S.; Li, X.; Wu, Z.-S.; Liang, J., Screen-printing fabrication of high volumetric energy density micro-supercapacitors based on high-resolution thixotropicternary hybrid interdigital micro-electrodes. Mater. Chem. Front. 2019, 3, 626-635.

(11)Shi, X.; Zhou, F.; Peng, J.; Wu, R. a.; Wu, Z. S.; Bao, X., One-Step Scalable Fabrication of Graphene-Integrated Micro-Supercapacitors with Remarkable Flexibility and Exceptional Performance Uniformity. Adv. Funct. Mater. 2019, 29, 1902860. 
(12)Zhou, Y.; Wang, X.; Acauan, L.; Kalfon-Cohen, E.; Ni, X.; Stein, Y.; Gleason, K. K.; Wardle, B. L., Ultrahigh-Areal-Capacitance Flexible Supercapacitor Electrodes Enabled by Conformal P3MT on Horizontally Aligned Carbon-Nanotube Arrays. $A d v$. Mater. 2019, 31, 1901916. 